\title{
Funktionelle kardiovaskuläre Störungen
}

\author{
Somatoforme, autonome Funktionsstörungen des kardiovaskulären \\ Systems - oft auch als "Herzneurose" oder "funktionelles kardiovaskuläres \\ Syndrom" bezeichnet - betreffen besonders jüngere Menschen vor dem \\ 40. Lebensjahr. Spezifische Kardiaka sind bei diesen Patienten nicht indi- \\ ziert. Sie brauchen vielmehr eine längerfristige Psychotherapie und \\ profitieren von naturheilkundlichen Verfahren.
}

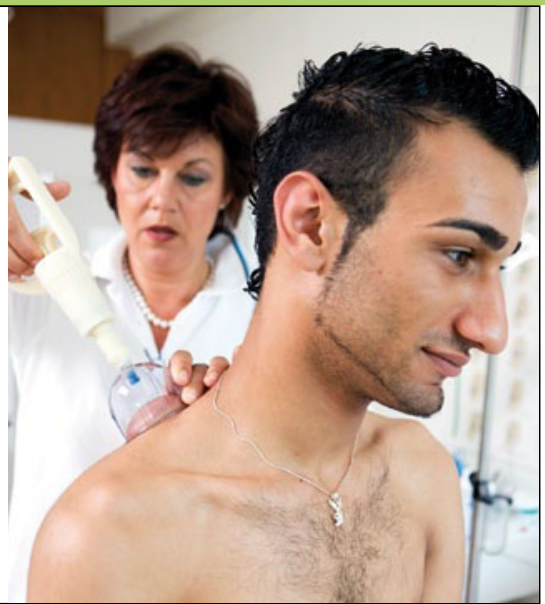

— Die üblichen organbezogenen Maßnahmen vermitteln Patienten mit funktionellen kardiovaskulären Störungen das Gefühl, schwer erkrankt zu sein und sind zudem nicht wirksam. Vielmehr ist eine längerfristig angelegte Psychotherapie zum Umgang mit Stress und Ängsten nötig. Parallel dazu müssen kurzfristig die akuten Symptome behandelt werden. Hierfür existieren viele naturheilkundliche Optionen.

Ergänzend zur Psychotherapie wird autogenes Training empfohlen. Man sollte mit der Wärme- und Atemübung beginnen und später die spezielle Herzübung verwenden. Auch die progressive Relaxation nach Jacobson eignet sich zur Linderung von Spannungszuständen und Unruhe.

\section{Schröpfen und \\ physikalische Therapie}

Bei vertebragener Symptomatik wird Schröpfen an den typischen Zonen des oberen oder mittleren Rückens (je nach Lokalbefund blutig oder trocken) empfohlen. Bei Dysfunktionen der mittleren BWS kann deren Beseitigung durch manuelle Medizin häufig zur Beschwerdefreiheit führen. Bei tachykarden Rhythmusstörungen sollte die obere BWS manualtherapeutisch behandelt werden.

Ziel der Verfahren aus der physikalischen Therapie ist die Harmonisierung der vegetativen Spannungslage. Tägliche Körpertrockenbürstungen und Wech- selduschen sind sehr zu empfehlen, außerdem ein wöchentlicher Saunabesuch. Bewegungstherapie wirkt dem Schonverhalten, das die meisten Patienten kennzeichnet, entgegen. Hier eignen sich v. a. Schwimmen, Ergometertraining und Arbeit an frischer Luft. Funktionelles Krafttraining sollte mit Ausdauersport kombiniert werden, wobei in der Regel drei Trainingseinheiten über 45-60 min/Woche ausreichen. Massagen (Bindegewebsmassage, Periosttherapie und Muskelmassage) eignen sich zur Reflexzonentherapie. Zudem empfehlen sich Luftbäder, Kneipp-Kuren und Klimakuren im Mittelgebirge.

\section{Phytotherapie}

Standardisierte Fertigarzneimittel aus dem Extrakt von Weißdornblättern und -blüten (300-900 mg pro Tag) fördern die Koronardurchblutung. Die Wirkung setzt erst nach einer mindestens vierwöchigen kontinuierlichen Einnahme ein. Extrakte aus Ginkgoblättern (120 mg/ Tag) unterstützen die periphere Durchblutung. Bei akuten Schwächeanfällen hat sich ein Kombinationspräparat aus Campher und Extrakt aus Weißdornfrüchten bewährt. Bei Druckgefühl im Oberbauch und reflektorischen Herzbeschwerden infolge von Luft im Magen oder Meteorismus mit Zwerchfellhochstand werden entblähend wirkende Phytotherapeutika eingesetzt, z. B. Tees aus Kümmel-, Anis- und Fenchelfrüchten.
Sedierend wirkende Phytotherapeutika wie Zubereitungen aus Melissenblättern und/oder Baldrianwurzel sind bei begleitenden Schlafstörungen und Unruhezuständen zu empfehlen.

\section{Herzsalben}

Eine segmentale Einreibung mit hautreizenden, hyperämisierend wirkenden Herzsalben, die der Patient selbst durchführen kann, hat sich gut bewährt. Sie enthalten ätherische Öle (Rosmarinoder Fichtennadelöl) und werden im Bereich der linken Thoraxhälfte bis zur Schulter und auf der Innenseite des linken Arms 2-3-mal täglich dünn aufgetragen. Da ätherische Öle gut in tiefere Hautschichten penetrieren, werden gleichzeitig Effekte auf oberflächliche und tiefere Gewebsstrukturen generiert und so die kutiviszeralen Reflexbögen intensiv aktiviert. Nach einer sechswöchigen Anwendung ist eine Therapiepause von ein bis zwei Wochen nötig, da es - wie bei allen Reiztherapien - zur Gewöhnung kommen kann. Aufgrund der Inhaltsstoffe sind bei Herzsalben Reizerscheinungen und Kontaktekzeme möglich. Durch die Inhaltsstoffe Campher, Menthol und Rosmarinöl können Überempfindlichkeitsreaktionen einschließlich Atemnot ausgelöst werden. Asthma bronchiale und Keuchhusten sind deshalb Kontraindikationen.

- Prof. Dr. med. Karin Kraft, Universität Rostock 